\title{
Searches for electroweak production of supersymmetric charginos, neutralinos and sleptons with the ATLAS detector
}

\section{Ryo Nagai*}

On behalf of the ATLAS Collaboration

Tokyo Institute of Technology

E-mail: nagai@hep.phys.titech.ac.jp

\begin{abstract}
Searches are presented for the direct production of charginos, neutralinos and sleptons in final states with at least one lepton and the missing transverse momentum. The analyses are based on approximate $20 \mathrm{fb}^{-1}$ of centre-of-mass energy of $\sqrt{s}=8 \mathrm{TeV}$ proton-proton collision data delivered by the Large Hadron Collider and recorded with the ATLAS detector in 2012. No significant deviation from the Standard Model expectation is observed. Exclusion limits are set at $95 \%$ confidence level in simplified models for each decay mode from electroweak production of supersymmetry.
\end{abstract}

XXII. International Workshop on Deep-Inelastic Scattering and Related Subjects, 28 April - 2 May 2014

Warsaw, Poland

\footnotetext{
* Speaker.
} 


\section{Introduction}

Supersymmetry (SUSY), which introduces a symmetry between bosons and fermions, is a promising candidate for beyond the Standard Model (SM). Each SM particle is required to have a super-partner which differs in the spin by a half from the SM particle. The super-partners of the electroweak gauge bosons $(W, Z, \gamma)$ are the electroweak gauginos $(\tilde{W}, \tilde{Z}, \tilde{\gamma})$, which are spin $1 / 2$ fermions. The electroweak gauginos mix with the SM higgs super-partners, higgsinos, and make up the mass eigenstates as charginos $\left(\tilde{\chi}_{i}^{ \pm}, i=1,2\right)$ and neutralinos $\left(\tilde{\chi}_{j}^{0}, j=1,2,3,4\right)$. On the other hand, the SM fermions such as leptons, neutrinos and quarks have spin 0 super-partners called sleptons $\tilde{\ell}$, sneutrinos $\tilde{v}$ and squarks $\tilde{q}$, respectively.

Theoretical motivations for SUSY are that it can provide a good candidate for the dark matter and solve the hierarchy problem in the gauge theory. In the assumption of $R$-parity conserving scenario, the lightest supersymmetric particle (LSP) should be stable, and if it is also neutral and weakly interacting, it is an excellent candidate for the dark matter. In the typical SUSY models such as the Minimal Supersymmetric Standard Model (MSSM), the LSP is the lightest neutralino, which contributes larger missing momentum than SM background at the Large Hadron Collider (LHC) [1].

During the LHC Run-1, searches for SUSY with multi-jets in final states have been carried out with great effort because high cross-section of the strong SUSY production is expected at the LHC and the super-partner of the top quark, stops $\tilde{t}$, should be light in the naturalness assumption. However, there have been no excess from the SM expectation in such modes and strong limits have already been set for the strong production of light coloured SUSY. Even though the electroweak production of SUSY has approximately three order of magnitude smaller cross-section than the strong production in the case of comparing them in the same mass scale, the direct production of electroweak SUSY may be dominant process if gluinos and squarks are massive. Thus the search for the electroweak SUSY is important in terms of a search for new physics in low energy scale. The data is used in these analyses that was recorded with the ATLAS detector [2] at the LHC in 2012 with the centre-of-mass energy of $\sqrt{s}=8 \mathrm{TeV}$ proton-proton collision corresponding to the integrated luminosity of approximate $20 \mathrm{fb}^{-1}$. The LSP $\tilde{\chi}_{1}^{0}$ is assumed to be the pure-bino $\tilde{B}^{0}$ and directly produced electroweak charginos $\tilde{\chi}_{1}^{ \pm}$and neutralinos $\tilde{\chi}_{2}^{0}$ are assumed to be the pure-wino $\tilde{W}^{ \pm}, \tilde{W}^{0}$ and to have degenerate mass spectra, $m_{\tilde{\chi}_{1}^{ \pm}}=m_{\tilde{\chi}_{2}^{0}}$. The branching ratio of the SUSY particle is assumed to be $100 \%$ in the selected modes (simplified models [3]).

Decay modes are assumed by the mass spectra of electroweak SUSY particles. For the direct electroweak gaugino production, two scenarios can be considered depending on the sleptons mass in the gauginos mass hierarchy. If sleptons are light, a produced gaugino can decay into a lepton (neutrino) and a slepton (sneutrino) which decay into a lepton and the LSP, as,

$$
\begin{gathered}
\tilde{\chi}_{1}^{ \pm} \rightarrow \ell^{ \pm} \tilde{v}\left(\text { or } v \tilde{\ell}^{ \pm}\right) \rightarrow \ell^{ \pm} v \tilde{\chi}_{1}^{0} \\
\tilde{\chi}_{2}^{0} \rightarrow \ell^{ \pm} \tilde{\ell}^{\mp}(\text { or } v \tilde{v}) \rightarrow \ell^{ \pm} \ell^{\mp} \tilde{\chi}_{1}^{0}\left(\text { or } v v \tilde{\chi}_{1}^{0}\right),
\end{gathered}
$$

where the masses of the sleptons and the sneutrinos are assumed to be $m_{\tilde{\ell}}=m_{\tilde{v}}=\left(m_{\tilde{\chi}_{1}^{ \pm}, \tilde{\chi}_{2}^{0}}+m_{\tilde{\chi}_{1}^{0}}\right) / 2$ (slepton-mediated scenario). The charginos $\tilde{\chi}_{1}^{ \pm}$and the neutralinos $\tilde{\chi}_{2}^{0}$ are assumed to decay into sleptons or sneutrinos with equal branching ratios for the lepton flavours. On the other hand, if all 
sleptons are heavier, a produced gaugino can decay into a boson and the LSP as,

$$
\begin{gathered}
\tilde{\chi}_{1}^{ \pm} \rightarrow W^{ \pm} \tilde{\chi}_{1}^{0} \rightarrow \ell^{ \pm} v \tilde{\chi}_{1}^{0} \\
\tilde{\chi}_{2}^{0} \rightarrow Z \tilde{\chi}_{1}^{0} \rightarrow \ell^{ \pm} \ell^{\mp} \tilde{\chi}_{1}^{0} \\
\tilde{\chi}_{2}^{0} \rightarrow h \tilde{\chi}_{1}^{0},
\end{gathered}
$$

where the mass of the SM higgs is assumed to be $125 \mathrm{GeV} / c^{2}$ (boson-mediated scenario).

For the direct slepton production, a produced slepton is simply assumed to decay directly into a lepton and the LSP as,

$$
\tilde{\ell}^{ \pm} \rightarrow \ell^{ \pm} \tilde{\chi}_{1}^{0}
$$

Considering these decay modes, there can be many leptons and less QCD contribution in final states for the electroweak SUSY production so that the signature should be clean. Therefore several signature based analyses were performed with two leptons [4], two taus [5], three leptons [6] and one lepton with two $b$-jets [7] in final states. More detail can be found in the corresponding references.

\section{Two leptons $(e, \mu)$ mode}

The analysis including two leptons $(e, \mu)$ in final states covers many production modes, such as $\tilde{\chi}_{1}^{ \pm} \tilde{\chi}_{1}^{\mp}, \tilde{\chi}_{1}^{ \pm} \tilde{\chi}_{2}^{0}$ and $\tilde{\ell} \tilde{\ell}$ direct production. In order to have exactly two leptons in final states, $\tilde{\chi}_{1}^{ \pm} \tilde{\chi}_{1}^{\mp}$ decaying via slepton-mediated and $W W$-mediated $\left(\tilde{\chi}_{1}^{ \pm} \tilde{\chi}_{1}^{\mp} \rightarrow W W \tilde{\chi}_{1}^{0} \tilde{\chi}_{1}^{0}\right)$ scenarios, $\tilde{\chi}_{1}^{ \pm} \tilde{\chi}_{2}^{0}$ decaying via $W Z$-mediated scenario $\tilde{\chi}_{1}^{ \pm} \tilde{\chi}_{2}^{0} \rightarrow W Z \tilde{\chi}_{1}^{0} \tilde{\chi}_{1}^{0} \rightarrow q q \ell \ell \tilde{\chi}_{1}^{0} \tilde{\chi}_{1}^{0}$ and direct slepton production scenario can be considered. For direct $\tilde{\chi}_{1}^{ \pm} \tilde{\chi}_{1}^{\mp}$ production scenarios, signal regions are defined with "stransverse mass" $m_{\mathrm{T} 2}$ [8] and $E_{\mathrm{T}}^{\text {miss,rel }}$, where $E_{\mathrm{T}}^{\text {miss,rel }}$ is defined from the missing transverse momentum $E_{\mathrm{T}}^{\mathrm{miss}}$ and the azimuthal angle between the $E_{\mathrm{T}}^{\mathrm{miss}}$ and the nearest lepton or jet $\Delta \phi_{\ell, j}$ as

$$
E_{\mathrm{T}}^{\mathrm{miss}, \text { rel }}=\left\{\begin{array}{ll}
E_{\mathrm{T}}^{\mathrm{miss}} & \text { if } \Delta \phi_{\ell, j} \geq \pi / 2 \\
E_{\mathrm{T}}^{\mathrm{miss}} \times \sin \Delta \phi_{\ell, j} & \text { if } \Delta \phi_{\ell, j}<\pi / 2
\end{array} .\right.
$$

The $E_{\mathrm{T}}^{\text {miss,rel }}$ can contribute to suppress events where the $E_{\mathrm{T}}^{\text {miss }}$ arises from significantly mis-measured leptons or jets. For direct $\tilde{\chi}_{1}^{ \pm} \tilde{\chi}_{2}^{0}$ production scenario, selection of the invariant masses for two jets in $W$ boson mass window and the same-flavour opposite-sign leptons pair in $Z$ boson mass window can strongly separate signal candidates from QCD $Z+$ jets background. For direct sleptons pair production scenario, signal regions are defined with $m_{\mathrm{T} 2}$ and requirement of opposite-sign leptons pair. These defined signal regions are not orthogonal so that the signal regions with the best expected exclusion limits for considered scenarios are chosen in the interpretations.

\section{Two taus mode}

The analysis including two taus in final states is a bit different from the strategy for two leptons mode though the target production modes are same as the two leptons. In the MSSM, a super-partner of a third generation SM particle might be light even if super-partners of the other 
generation SM particles are massive. Thus it is important to search signals with taus in final states even though identification and optimisation of taus are difficult. In order to avoid signature overlap with the other modes such as two $e, \mu$ and three leptons, events containing $e$ or $\mu$ are vetoed. This makes it possible to keep all signal regions orthogonal and to combine them.

The dominant background for this mode is the fake taus contribution. It is hard to estimate the fake contribution with Monte Carlo simulations because of difficulty of theoretical QCD modeling so that a data-driven method called "ABCD method" is applied.

\section{Three leptons $(e, \mu, \tau)$ mode}

The analysis including three leptons $(e, \mu, \tau)$ in final states is performed to focus on $\tilde{\chi}_{1}^{ \pm} \tilde{\chi}_{2}^{0}$ production mode. The cross-section of this channel is predicted to be the largest among the electroweak SUSY production processes. If both $\tilde{\chi}_{1}^{ \pm}$and $\tilde{\chi}_{2}^{0}$ decay leptonically, the events should include three leptons in final states. Therefore this analysis can cover many decay modes. Four kinds of scenarios are considered, decay via slepton-mediated, stau-mediated, $W Z$-mediated and $W h$-mediated scenarios. The analyses for slepton-mediated and $W Z$-mediated scenarios are optimised without taus to make 20 bins in three variables: $E_{\mathrm{T}}^{\text {miss }}$, the invariant mass of the same-flavour opposite-sign lepton pair closest to the $Z$ boson mass $m_{\mathrm{SFOS}}$, and the transverse mass $m_{\mathrm{T}}$ for the $E_{\mathrm{T}}^{\text {miss }}$ and a lepton which is not used to reconstruct $m_{\mathrm{SFOS}}$. For stau-mediated scenario, a signal region is optimised using two taus with high $E_{\mathrm{T}}^{\mathrm{miss}}$ and the maximum "stransverse mass" $m_{\mathrm{T} 2}^{\max }$. For $W h$-mediated scenario, multiple disjoint signal regions are defined by the target decay modes of the SM higgs, $h \rightarrow W W$ and $h \rightarrow \tau \tau$. For $h \rightarrow W W$ mode, requirement on no taus and the minimum azimuthal angle between two opposite-sign leptons $\Delta \phi_{\ell \ell^{\prime}}^{\min }$ can suppress the background. Signal regions for $h \rightarrow \tau \tau$ mode are optimised using at least one tau in final states with high $p_{\mathrm{T}}$ from taus. An additional requirement is available for two taus signal regions that the invariant mass of opposite-sign taus pair $m_{\tau \tau}$ should be consistent with $h \rightarrow \tau \tau$ decay. All signal regions except two taus signal regions are orthogonal so that they can be statistically combined, where two taus signal regions are selected based on the best expected limits.

The dominant background processes are dibosons $(W Z, Z Z)$ and $t \bar{t}$ productions. Reducible background such as $t \bar{t}$ production is estimated by a data-driven method called "matrix method" in each signal region.

\section{One lepton and two $b$-jets mode}

The target of this analysis including one lepton and two $b$-jets in final states is direct production of $\tilde{\chi}_{1}^{ \pm} \tilde{\chi}_{2}^{0}$ decaying via $W h$-mediated scenario, where the SM higgs decays into $b$-jets pair: $\tilde{\chi}_{1}^{ \pm} \tilde{\chi}_{2}^{0} \rightarrow$ $W h \tilde{\chi}_{1}^{0} \tilde{\chi}_{1}^{0} \rightarrow \ell v b \bar{b} \tilde{\chi}_{1}^{0} \tilde{\chi}_{1}^{0}$. The branching ratio of $h \rightarrow b \bar{b}$ is approximately $60 \%$, which is the largest branching ratio in all of the SM higgs decay modes. The requirements for the optimisation of this analysis are applied that one hard lepton should pass the single lepton trigger and the invariant mass of two $b$-tagged [9] jets $m_{b b}$ should be in the SM higgs mass window, $105-135 \mathrm{GeV} / c^{2}$. Further selection is applied with the $E_{\mathrm{T}}^{\text {miss }}$, the transverse mass for the lepton $m_{\mathrm{T}}$ and the "contransverse mass" $m_{\mathrm{CT}}[10]$ for two $b$-jets, which is expressed as

$$
m_{\mathrm{CT}}^{2}=\left(E_{\mathrm{T}}^{b_{1}}+E_{\mathrm{T}}^{b_{2}}\right)^{2}-\left|\mathbf{p}_{\mathrm{T}}^{b_{1}}-\mathbf{p}_{\mathrm{T}}^{b_{2}}\right|^{2} .
$$


Background estimation is implemented by the simultaneous background only fit using $m_{b b}$ bins in the side bands of control regions.

\section{Results}

The number of observed events is consistent with the SM expectation in all signal regions within statistic and systematic uncertainties. Figures 1 show the typical distributions for two and three leptons modes, in which the consistency with SM expectation can be seen clearly. The exclusion limits are set in the simplified models for all scenarios covered by the analyses. Figures 2 show the expected and observed exclusion limits for each process, where the limits are set at $95 \%$ confidence level using the $\mathrm{CL}_{s}$ prescription [11]. For direct stau pair production scenario, the observed excluded cross-section is approximately $0.17 \mathrm{pb}$ by two taus mode but there are no observed exclusion limits at $95 \%$ confidence level due to the low cross-section and low sensitivity for this scenario.

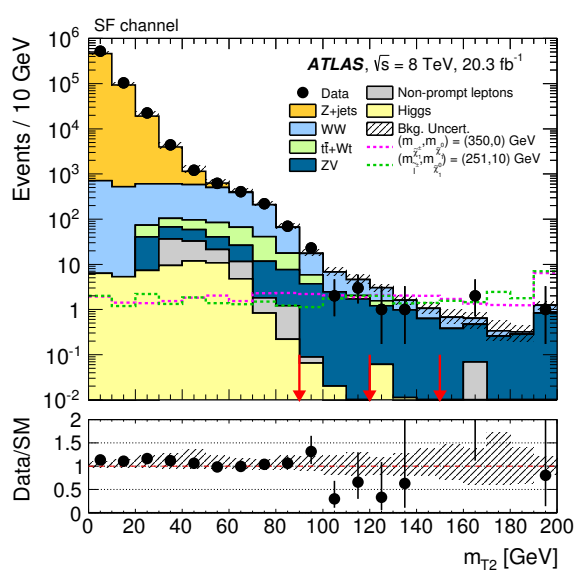

(a) Distribution of $m_{\mathrm{T} 2}$ for two leptons mode

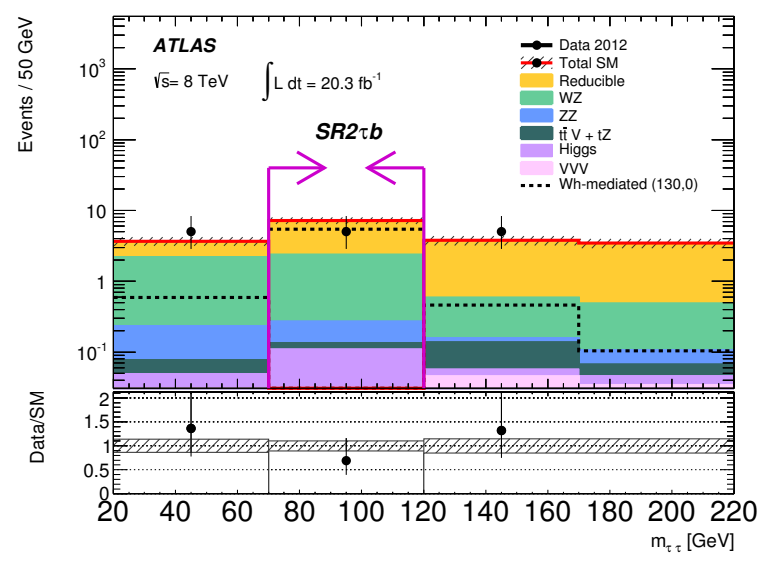

(b) Distribution of $m_{\tau \tau}$ for three leptons mode

Figure 1: Typical distributions from two and three leptons modes. The $m_{\mathrm{T} 2}$ in the same-flavour two leptons is shown in (a) and the $m_{\tau \tau}$ reconstructed by the opposite-sign two taus in the events including additionally one hard lepton is shown in (b). There is no significant deviation from SM expectation for each scenario.

\section{Conclusion}

Searches for electroweak production of supersymmetric particles with the ATLAS detector are presented. The consideration for electroweak SUSY production is now very important in two reasons. One is that the LSP can be the lightest neutralino in the MSSM, which is a good candidate for the predicted dark matter. Another is that strong production of light SUSY is already excluded so that the only way to search for light SUSY is electroweak production. Several kinds of production modes by the combination of charginos, neutralinos or sleptons can be considered. In addition, direct charginos or neutralinos production modes can be divided by the mass hierarchy of sleptons. If sleptons are light, assuming $m_{\tilde{\ell}}=\left(m_{\tilde{\chi}_{1}^{ \pm}, \tilde{\chi}_{2}^{0}}+m_{\tilde{\chi}_{1}^{0}}\right) / 2$, charginos and neutralinos decay via slepton into the LSPs. Otherwise they decay via bosons $(W, Z, h)$ into the LSPs. No significant 


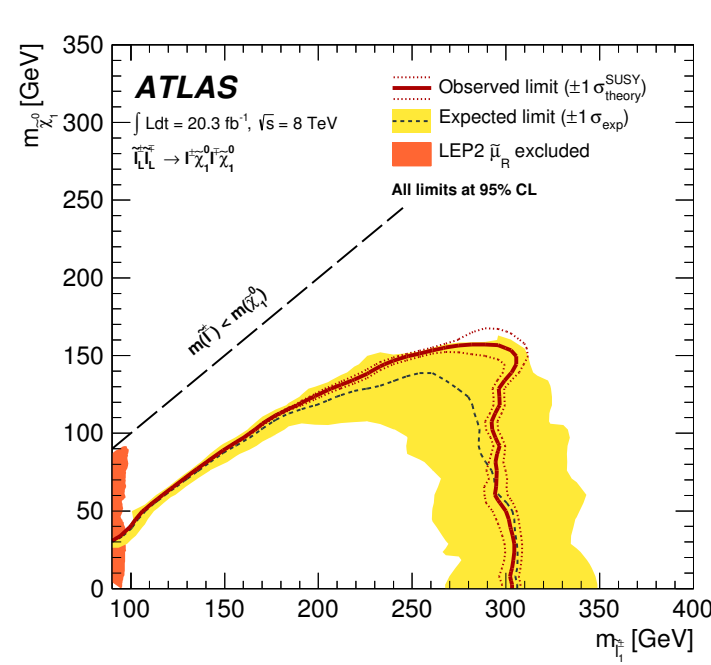

(a) Direct slepton production

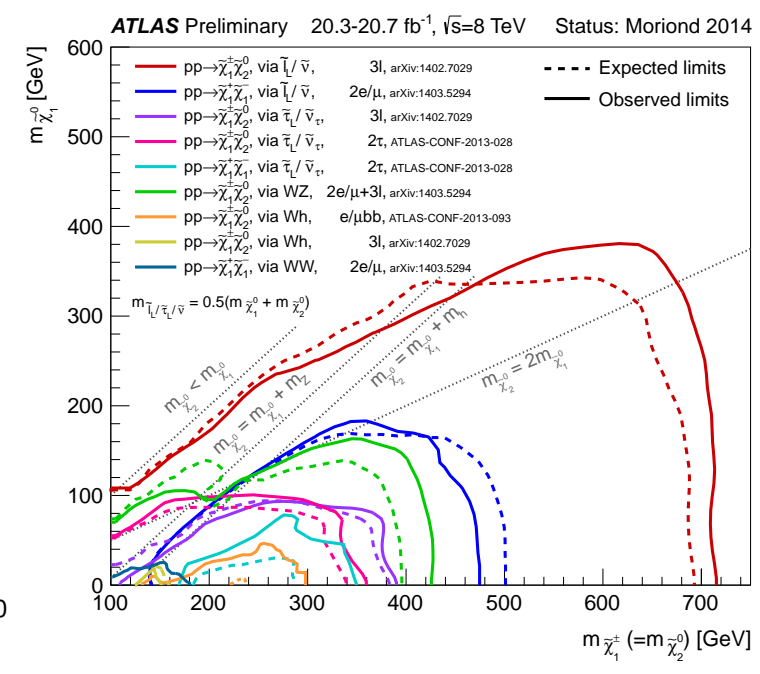

(b) Direct $\tilde{\chi}_{1}^{ \pm}$or $\tilde{\chi}_{2}^{0}$ production [12]

Figure 2: Expected and observed exclusion limits for electroweak SUSY production scenarios, direct slepton production in (a) and direct charginos or neutralinos production in (b) [12]. These figures cover all of the limits set by the analyses at $95 \%$ confidence level.

excess is observed and the distributions are consistent with the SM expectation. Observed exclusion limits are set in simplified models for the electroweak SUSY production modes. Exclusion limits for phenomenological Minimal Supersymmetric Standard Models (pMSSM) are also set in the corresponding references $[4,5,6]$.

\section{References}

[1] L. Evans and P. Bryant, 2008 JINST 3 S08001.

[2] ATLAS Collaboration, 2008 JINST 3 S08003.

[3] J. Alwall et al., Phys. Rev. D 79 (2009) 075020.

[4] ATLAS Collaboration, JHEP 05 (2014) 072, arXiv:1403.5294.

[5] ATLAS Collaboration, ATLAS-CONF-2013-028, https://cds.cern.ch/record/1525889.

[6] ATLAS Collaboration, JHEP 04 (2014) 169, arXiv:1402.7029.

[7] ATLAS Collaboration, ATLAS-CONF-2013-093, https://cds.cern.ch/record/1595756.

[8] A. Barr, C. Lester and P. Stephens, J. Phys. G29 (2003) 2343-2363.

C. Lester and D. Summers, Phys. Lett. B463 (1999) 99-103.

[9] ATLAS Collaboration, ATLAS-CONF-2011-102, https://cds.cern.ch/record/1369219.

[10] D. Tovey, JHEP 0804 (2008) 034.

G. Polesello and D. Tovey, JHEP 1003 (2010) 030.

[11] A. L. Read, J. Phys. G 28 (2002) 2693-2704.

[12] ATLAS Collaboration, ATLAS public web pages, https://atlas.web.cern.ch/Atlas/ GROUPS/PHYSICS/CombinedSummaryPlots/SUSY/index.html. 